title:

\title{
Chemistry meets epigenetics: from chromatin tools to small molecules affecting the readout of our genomes
}

The functional state of eukaryotic genomes is regulated by sequence-specific DNA binding factors and by the packaging state of DNA into chromatin. Dissection of the complex crosstalk of gene regulation and cellular inputs has been substantially aided by the development of chemical tools targeting the various factors and different components involved in these processes.

The term 'Chemical Genetics' was coined in the 1990ies emphasizing the concept of chemical compounds that emulate the effects of genetic manipulations.[1] Besides genetic information, which is stored in the sequence of DNA, epigenetic phenomena constitute an essential layer of genome regulation. On a molecular level, epigenetic changes are manifested by chemical modifications of nucleic acids and of the packaging proteins of eukaryotic genomes - the histones. The key players directing epigenetic genome regulation are factors that install, remove, or bind to the chemical modifications of nucleic acids or histone proteins. These are commonly referred to as writers, erasures and readers. Discovering the substrate sites and control mechanisms of these proteins as well as uncovering the functional consequences of epigenetic genome modifications is a central challenge of Chemical Epigenetics. Moreover, the readers, writers and erasers provide new opportunities for drug discovery because these are often dysregulated in diseases like cancer. Major efforts in medicinal chemistry have identified small molecule ligands and natural products targeting these proteins. The various drugs serve as tools for basic research and are finding their way into the clinics. The fact that natural and man-made compounds affect the modification status of nucleic acids and histone proteins gave rise to a new research field, environmental epigenetics. While the epigenetic chromatin modifications are generally reversible, there is an inheritable aspect to these changes. Indeed, epigenetic traits that were acquired on the basis of exposure of organisms to environmental toxins, for example via the diet, can be passed to following generations. The discovery of environmental epigenetics has changed the traditional picture of inheritance and might be the key for a comprehensive understanding of developmental processes in health and disease.

We focused the section of Chemical Genetics of this current issue of Current Opinion in Chemical Biology on Chemical Epigenetics by highlighting recent advances of this vibrant research field in the following areas: (1) epigenetic histone modifications, (2) epigenetic modifications of nucleic acids, (3) small molecule modulators of epigenetic writers, erasers and readers and (4) environmental epigenetics.

The review article by Davis and colleagues introduces the field of posttranslational modifications (PTMs) of histones that are a central means of epigenetic gene regulation. PTMs are primarily installed at the $\mathrm{N}$-terminal histone tail regions, which are protruding outwards of the fundamental building blocks of chromatin, the nucleosomes. There they serve as binding sites for chromatin factors and modulate chromatin structure. The 'histone code theory' considers histone tails as a kind of programming platform of epigenetic information that is deposited in form of PTMs.[2] The article provides a comprehensive overview of chemical strategies for identifying histone PTMs and for introducing PTMs into histones. It also discusses how the chemical biology approaches have allowed new insights into chromatin function and activity. The selective introduction of histone PTMs is a major challenge for chromatin research. Chemical biology has been shaping the field with the development of strategies such as protein semisynthesis for site-specific protein modification. The contribution by Chatterjee and colleagues highlights recent advances in the seminsynthesis of histones and their use for investigating the histone code. The article also emphasizes the cross-talk of 
two prominent histone PTMs: methylation and ubiquitylation. Lysine-ubiquitylation and the related SUMOylation is unique among the histone PTMs because this modification is not a small chemical group but addition of a small protein linked to a lysine side chain by an isopeptide bond. Lysine-ubiquitylation of histones plays important roles in transcription, replication and DNA repair. The site-specific installation of this PTM requires elaborated strategies involving semi and total synthesis that are comprehensively discussed in the article by Brik and colleagues. The authors also provide a detailed overview of the use of synthetic ubiquitylated histones in chromatin research. Besides protein semisynthesis, amber suppression technology has emerged as a highly potent approach for installing PTMs into histones. The article by Neumann, Summerer and colleagues describes recent advances and applications of this technology for chromatin research. The article also highlights related approaches for mapping the recruitment of factors to chromatin modifications in vivo by using genetically encoded photo-crosslinkers.

Besides histone PTM, chemical modifications of the DNA nucleobases are central means of epigenetic gene regulation. Of major interest in this regard is 5 -methylcytosine $(5 \mathrm{mC})$, a modification that mediates gene silencing and that is passed site-specifically from one cell generation to the next thereby serving as an inheritable mark. A set of a-ketoglutaratedependent dioxygenases, referred to as TET enzymes can oxidize $5 \mathrm{mC}$. This process is not only important in DNA demethylation but the oxidized $5 \mathrm{mC}$ bases also have independent signaling function. The contribution by Kohli and colleagues discusses promising approaches of site-specific genomic editing of DNA modifications by engineering the DNA modifying enzymes. Modifications of nucleobases are not restricted to DNA but have also been identified in RNA. The role of such RNA modifications is currently under intensive investigation and constitutes the basis of 'epitranscriptomics'. The review by Yi and colleagues describes recent advances in this new field with a focus on discovery and function of N1-methyladenosine in non-coding and messenger RNAs.

The writers, erases and readers play a central role in epigenetic gene regulation because their balanced action controls global and local modification states of chromatin. The development of inhibitors and small molecule ligands targeting these enzymes has been instrumental for epigenetic research and provides modern medicine with new drugs addressing aberrant gene expression. Among the many chromatin modifications, methylation stands out in terms of versatility. Besides $5 \mathrm{mC}$ in DNA and N1-methyladenosine in RNA, various lysine and arginine residues in histones are methylated to various degrees (i.e. mono-, di-, trimethylation). The article by Jung and colleagues provides a comprehensive review of recent developments in the field of small molecule modulators of histone and DNA methylation as well as the enzymes involved in this process. A further key modification of histones is lysine acetylation. This modification is associated with active transcription and is highly abundant in histones and also non-histone proteins. The contribution by Mapp and colleagues focuses on two master coactivators, CBP and p300. As these are involved in the regulation of many genes, intensive efforts have searched for chemical modulators of these lysine acetyl-transferases. The article summarizes recent successes in the development of chemical tools selectively targeting several CBP/p300 domains and their application in fostering our understanding of the regulation of these factors. Besides the modifying enzymes, the readers of chromatin modifications have entered the center stage as targets for drug discovery. The review by Conway and colleagues addresses recent drug discovery efforts targeting bromodomains as major readers of histone lysine acetylation. The article further discusses promising advances in the development of inhibitors of writers and erasers of histone acetylation, highlighting the chances that epigenetic drugs provide for modern medicine.

Differences in chromatin architecture are at the core of the distinct gene expression profiles that are establishing and manifesting the identity of distinct cell types in multicellular organisms. It is emerging that environmental conditions such as the presence of toxins, diet, temperature, maternal behavior and other external factors can result in sustained differences in the DNA and histone modification landscape and thereby epigenetic state of cells and 
organisms. On the example of the human brain that is constituted of a staggering number of different cell types, Barco and colleagues review recent studies that identify and demonstrate the existence of environmental fingerprints that shape the neuronal epigenome. Their article focuses on the role of exposure to adverse environmental factors has in the occurrence of neurological diseases and discusses the major challenges this important research field is facing for clarifying mechanisms of pathoethiology and for uncovering new strategies of treatment. While research on the human environmental epigenome faces special challenges, studies in model organisms such as mice have established causality between exposure to chemical and environmental agents and molecular chromatin changes. On the example of imprinted genes and endogenous retroviruses Feil and colleagues discuss how various environmental factors can perturb epigenetic regulatory processes. Although the exact molecular mechanisms are still poorly understood, changes in DNA methylation patterns appear central in this process. In this context, Meehan and colleagues describe how DNA modification 'barcoding' of $5 \mathrm{mC}$ and its oxidation product 5 -hydroxymethylcytosine $(5 \mathrm{hmC})$ can be used in risk assessment of dose response, temporal and transient exposure of organisms to xenobiotics. They propose that such signatures are unique to a given drug exposure/disease state and that these can potentially be predictive of downstream consequences.

We hope that you will find this section on Chemical Epigenetics in Current Opinion in Chemical Biology informative and stimulating for your research. We certainly have learned a lot in editing this section and are anticipating many new insights to originate from the application of chemical biology to epigenetics research in the next few years.

Dirk Schwarzer and Wolfgang Fischle

\section{References:}

1. Schreiber SL: Chemical genetics resulting from a passion for synthetic organic chemistry. Bioorganic \& Medicinal Chemistry 1998, 6:1127-1152.

2. Strahl BD, Allis CD: The language of covalent histone modifications. Nature 2000, 403:41-45.

\section{biography WF:}

Wolfgang Fischle studied biochemistry at the University of Tübingen. For his PhD studies he joined the laboratory of Eric Verdin at the J.D. Gladstone Institutes. After postdoctoral work with David Allis at The Rockefeller University, he was appointed Max Planck Research Group Leader at the MPI-bpc in Göttingen. In 2015 he was appointed Professor of Bioscience at King Abdullah University of Science and Technolopgy (KAUST).

His research interests are focused on the molecular analysis of chromatin regulatory processes.

\section{biography DS:}

Dirk Schwarzer studied chemistry at the University of Marburg and completed his PhD with Mohamed A. Marahiel. After post-doctoral research with Philip A. Cole at the Johns-Hopkins University, he established his own group at the Leibniz-Insititut für Molekulare Pharmakologie (FMP) in Berlin. In late 2011 he was appointed Professor of Biochemistry at the University of Tübingen and joined the Interfaculty Institute of Biochemistry.

His research interests focus on chemical tools for posttranslational protein modifications. 
